\title{
Effect of back reaction on light quark energy loss in heavy quark cloud
}

\author{
Zi-qiang Zhang ${ }^{1,2, a}$, De-fu Hou ${ }^{2, b}$ \\ ${ }^{1}$ School of Mathematics and Physics, China University of Geosciences, Wuhan 430074, China \\ ${ }^{2}$ Key Laboratory of Quark and Lepton Physics (MOE) and Institute of Particle Physics, Central China Normal University, Wuhan 430079, China
}

Received: 7 April 2020 / Accepted: 13 October 2020 / Published online: 27 October 2020

(C) The Author(s) 2020

\begin{abstract}
We study the effect of back reaction on the energy loss of light quarks in strongly coupled $\mathcal{N}=4$ supersymmetric Yang-Mills (SYM) plasma, by using the AdS/CFT correspondence. We perform the analysis within falling string and shooting string approaches, respectively. It is shown that the back reaction, arising from the presence of static heavy quarks uniformly distributed over SYM, enhances the energy loss, in agreement with the findings of the drag force and jet quenching parameter.
\end{abstract}

\section{Introduction}

It has been widely accepted that the ultra-relativistic nucleusnucleus collisions at the Relativistic Heavy-Ion Collider (RHIC) and Large Hadron Collider (LHC) have created a new type of matter, called quark-gluon plasma (QGP). One of the striking features of QGP is jet quenching: when energetic jet partons pass through QGP, they interact with it and thus lose their energy (for recent reviews of jet quenching, see for example [1,2]). On the other hand, a lot of experimental research indicates that QGP behaves as a strongly coupled, near-perfect fluid $[3,4]$. Therefore, it is of great interest to study the jet quenching in strongly coupled settings.

In this regard, the conjectured AdS/CFT duality between type IIB string theory, formulated on $\operatorname{AdS}_{5} \times S^{5}$ and $\mathcal{N}=4$ SYM theories in four dimensions [5-7], provides a powerful tool for studying various aspects of QGP (see [8] for a recent review with many phenomenological applications). Already, the jet quenching of heavy quarks traversing in $\mathcal{N}=4 \mathrm{SYM}$ plasma has been studied by the drag force extracted from a trailing string moving in the dual geometry $[9,10]$. On the other hand, the energy loss of light quarks traveling through the same plasma has been analyzed in various ways, such as the jet quenching parameter [11,12], falling string [13-17], and shooting string $[18,19]$ approaches.

The purpose of this paper is to study the effect of back reaction on the energy loss of light quarks. As is well known, the QGP is comprised of a large amount of free quarks and gluons. That would mean that, if one analyzes the dynamics of a quark, the back reaction of other quarks may be taken into account. But as far as we know, it remains difficult to conduct such a research by considering the back reacting of the string on the plasma (see [20] for some progress in this direction). However, Chakrabortty recently proposed [21] a relatively simple back reacted geometry, parameterized by the mass of the black hole and long string density. Specifically, one considers a uniform distribution of infinitely long, static strings hanging from the boundary of the AdS black hole and stretching up to the horizon. Due to the presence of these long strings, the AdS black hole gets back reacted [21-25]. Later, the drag force [21] and jet quenching parameter [22] have been discussed in such a theory. It was found that the inclusion of back reaction increases the drag force and jet quenching with the canonical definition of the energy scale. The absence of local physics makes it difficult to use a parameter thus enhancing the energy loss. We then ask: how does such back reaction modify the light quark energy loss? Does such a back reaction have the same effect on the energy loss of light quarks as with heavy quarks? We shall attempt to answer these questions in the present work.

The structure of the paper is as follows. In the next section, we introduce the back reacted gravity background given in [21]. In Sects. 3 and 4, we study the effect of back reaction on the energy loss of light quarks within the falling string and shooting string approaches, in turn. Finally, we conclude with the significance of our results in Sect. 4.

\footnotetext{
a e-mail: zhangzq@cug.edu.cn

b e-mail: houdf@mail.ccnu.edu.cn
} 


\section{Setup}

The effect of back reaction could be described by the following gravitational action [21]:

$S=\frac{1}{16 \pi G_{5}} \int d^{5} x \sqrt{-g}(\mathcal{R}-2 \Lambda)+S_{m}$,

where $G_{5}$ is the 5 -dimensional Newton constant. $\mathcal{R}$ denotes the Ricci scalar. $\Lambda$ represents the cosmological constant. $S_{m}$ is the matter part of the action, given by

$S_{m}=-\frac{1}{2} \sum_{i} \mathcal{T}_{i} \int d^{2} \epsilon \sqrt{-h} h^{\alpha \beta} \partial_{\alpha} X^{\mu} \partial_{\beta} X^{v} g_{\mu \nu}$,

where $\mathcal{T}_{i}$ is the tension. $h^{\alpha \beta}$ and $g^{\mu \nu}$ are the world-sheet metric and space-time metric, respectively. $\alpha, \beta$ are the world sheet coordinates. $\mu, v$ are the space-time directions.

The equations of motion with respect to the action (1) are

$\mathcal{R}_{\mu \nu}-\frac{1}{2} \mathcal{R} g_{\mu \nu}+\Lambda g_{\mu \nu}=8 \pi G_{5} T_{\mu \nu}$,

with

$$
\begin{aligned}
T_{\mu \nu}= & -\sum_{i} \mathcal{T}_{i} \int d^{2} \epsilon \frac{1}{\sqrt{\left|g_{\mu \nu}\right|}} \\
& \sqrt{\left|h_{\alpha \beta}\right|} h^{\alpha \beta} \partial_{\alpha} X^{\mu} \partial_{\beta} X^{v} \delta_{i}^{5}(x-X),
\end{aligned}
$$

where the delta function denotes the source divergences due to the presence of the strings.

Following [21], one considers the space-time as

$\mathrm{d} s^{2}=g_{t t}(r) \mathrm{d} t^{2}+g_{r r}(r) \mathrm{d} r^{2}+r^{2} \delta_{a b} \mathrm{~d} x^{a} \mathrm{~d} x^{b}$,

where $(\mathrm{a}, \mathrm{b})$ run over $\mathrm{n}-1$ space directions.

Using the static gauge $t=\xi^{0}, r=\xi^{1}$, the non-vanishing components of $T^{\mu \nu}$ become

$T^{t t}=-\frac{b g^{t t}}{r^{n-1}}, \quad T^{r r}=-\frac{b g^{r r}}{r^{n-1}}$,

where the strings are considered to be uniformly distributed over $n-1$ directions with the density

$b(x)=T \sum_{i} \delta_{i}^{(n-1)}\left(x-X_{i}\right), \quad$ with $\quad b>0$.

By solving the Einstein equation, one has

$V(r)=K+\frac{r^{2}}{R^{2}}-\frac{2 m}{r^{n-1}}-\frac{2 b}{(n-1) r^{n-3}}$,

where $K=0,-1,1$ depending on whether the boundary is flat, spherical or hyperbolic, respectively; and $R$ denotes the AdS radius. In this work we are mostly interested in the case of $K=0, n=4$. Given that, the metric reads

$\mathrm{d} s^{2}=\frac{r^{2}}{R^{2}}\left(-f(r) \mathrm{d} t^{2}+\mathrm{d} \vec{x}^{2}\right)+\frac{R^{2}}{r^{2} f(r)} \mathrm{d} r^{2}$, with

$f(r)=1-\frac{2 m R^{2}}{r^{4}}-\frac{2}{3} \frac{b R^{2}}{r^{3}}$,

where $r$ denotes the coordinate describing the 5th dimension. The boundary is $r=\infty$. The horizon is located at $r=r_{h}$, defined by $f\left(r_{h}\right)=0$. This allows us to write $m$ as

$m=\left(1-\frac{2}{3} \frac{b R^{2}}{r_{h}^{3}}\right) \frac{r_{h}^{4}}{2 R^{2}}$.

The temperature of the black hole is

$T=\left.\frac{\sqrt{g^{r r}} \partial_{r} \sqrt{g_{t t}}}{2 \pi}\right|_{r=r_{h}}=\frac{6 r_{h}^{3}-b R^{2}}{6 \pi R^{2} r_{h}^{2}}$.

On the other hand, if one works with $z=R^{2} / r$ as the radial coordinate, then (9) turns into

$\mathrm{d} s^{2}=\frac{R^{2}}{z^{2}}\left(-f(z) \mathrm{d} t^{2}+\mathrm{d} \vec{x}^{2}+\frac{\mathrm{d} z^{2}}{f(z)}\right)$,

with

$f(z)=1-\frac{2 m z^{4}}{R^{6}}-\frac{2}{3} \frac{b z^{3}}{R^{4}}$,

now the horizon is $z=z_{h}$ and the boundary is $z=0$.

It should be noted that (9) or (13) is thermodynamically stable under tensor and vector perturbations. Also, it recovers to usual $A d S_{5}$-Schwarzschild black hole for $b=0$. For more details, we refer to [21].

\section{Back reaction effect on light quark energy loss}

\subsection{Falling string}

In AdS/CFT, a possible way to study the energy loss of light quarks is using a falling string. As in previous studies, various authors [13-17] have used this method to study the energy loss of massless, high-energy jets passing through $\mathcal{N}=4$ SYM plasma in different ways, e.g., with or without the addition of fundamental-charge matter and specifying different initial conditions. Interestingly, almost all of the analyses with respect to the stopping distance traveled by the falling string in $A d S_{5}$-Schwarzschild black hole are consistent. Here we employ the approach of [16,17], where an R-charged current is generated by a massless gauge field in the gravity dual. The induced current is considered as an energetic jet passing through the medium. When the wave packet of the massless gauge field falls into the horizon of the dual geometry, the image jet on the boundary dissipates and thermalizes (in the medium). The stopping distance is therefore defined as the distance for a jet passing through the medium before it thermalizes. 
In the WKB approximation, the wave packet of the gauge field in the dual geometry is highly localized in the momentum space. Given that, the wave function of the gauge field can be factorized as

$$
A_{j}(t, z)=\exp \left[\frac{i}{\hbar}\left(q_{k} x^{k}+\int \mathrm{d} z q_{z}\right)\right] \tilde{A}_{j}(t, z),
$$

where $q_{k}$ is the 4-momentum, conserved as the metric preserves the translational symmetry along the 4-dimensional space-time. $q_{z}$ denotes the momentum along the bulk direction. $\tilde{A}_{j}(t, z)$ represents the slow-varying with respect to $t$ and $z . j, k$ are the 4-dimensional space-time coordinates.

In the classical limit (or $\hbar \rightarrow 0$ ), the equation of motion of the wave pack will reduce to a null geodesic,

$0=\left(\mathrm{d} s^{2}\right)=\mathrm{d} x^{i} g_{i j} \mathrm{~d} x^{j}+\mathrm{d} z g_{z z} \mathrm{~d} z$,

resulting in

$$
\frac{\mathrm{d} z}{\mathrm{~d} \lambda}=\frac{1}{\sqrt{g_{z z}}}\left[-g_{i j} \frac{\mathrm{d} x^{i}}{\mathrm{~d} \lambda} \frac{\mathrm{d} x^{j}}{\mathrm{~d} \lambda}\right]^{1 / 2},
$$

where $\lambda$ is an affine parameter for the trajectory. The 4dimensional translation invariance,

$g_{i j} \frac{\mathrm{d} x^{j}}{\mathrm{~d} \lambda}$

is conserved and proportional to $q_{i}$, yielding

$$
\frac{\mathrm{d} x^{i}}{\mathrm{~d} \lambda} \propto g^{i j} q_{j} .
$$

Then dividing (19) by (17) gives

$$
\frac{\mathrm{d} x^{i}}{\mathrm{~d} z}=\sqrt{g_{z z}} \frac{g^{i j} q_{j}}{\left(-q_{k} g^{k l} q_{l}\right)^{1 / 2}} .
$$

To proceed, one assumes the 3-momentum $\vec{q}$ to point in one of $\vec{x}$ directions, say the $x_{3}$ direction, and writes $q_{i}=(-\omega, 0,0,|\vec{q}|)$, where $\omega$ and $\vec{q}$ are the energy and the spatial momentum of the particle, respectively. Then from (13) and (20), the stopping distance for the back reacted gravity background is obtained:

$x=\int_{0}^{z_{h}} \frac{\mathrm{d} z}{\left(1-\frac{q^{2}}{|\vec{q}|^{2}}-f(z)\right)^{1 / 2}}$,

where $q^{2} \equiv q_{i} \eta^{i j} q_{j}=-\omega^{2}+|\vec{q}|^{2}$.

In order to compare with [17], we work with the coordinate $u \equiv z^{2} / 4$ instead of $z$. Then (21) becomes

$$
x=\int_{0}^{u_{h}} \frac{\mathrm{d} u}{\sqrt{u\left(\frac{u^{2}}{u_{h}^{2}}-\frac{q^{2}}{|\vec{q}|^{2}}-\frac{16 b u^{3 / 2}}{3}\left(\sqrt{\frac{u}{u_{h}}}-1\right)\right)}} .
$$

Note that by setting $b=0$ and $u_{h}=1$ (which corresponds to $2 \pi T=1$ ) in (22), the stopping distance of SYM

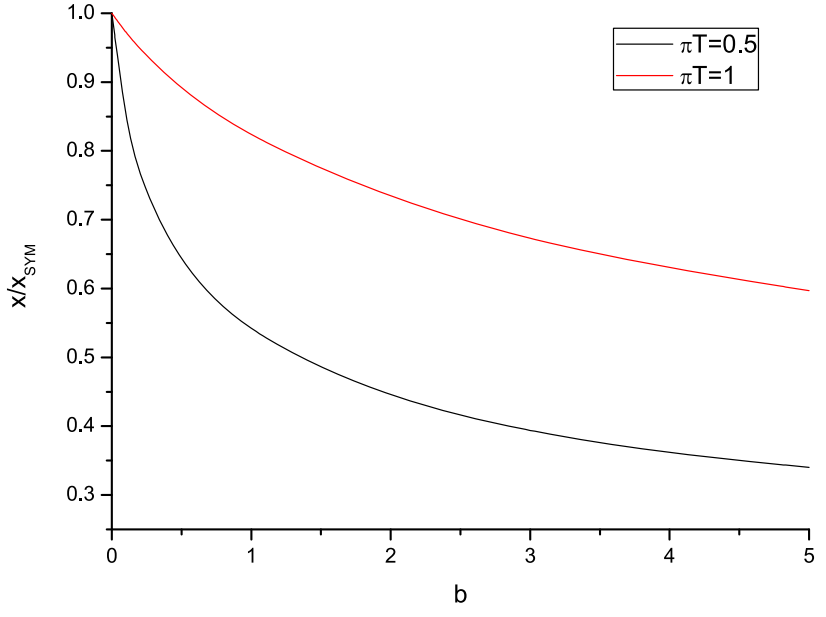

Fig. 1 Left: $x / x_{\text {SYM }}$ versus $b$ with fixed $T$. Here we take $|\vec{q}|=0.99 \omega$ and $R=1$

is reproduced, that is,

$x_{\mathrm{SYM}}=\int_{0}^{1} \frac{u}{\sqrt{u\left(u^{2}-\frac{q^{2}}{|\vec{q}|^{2}}\right)}}$,

which is exactly Eq. (2.3) in [17]. We tried to derive the analytic expression for (22) to see how the stopping distance scales with the jet's energy for a given value of $b$ but have not succeeded yet, so we have to turn to numerics.

In Fig. 1, we compare the stopping distance in the medium with and without back reaction for two different temperatures, where the top is for $\pi T=1$, while the bottom is for $\pi T=0.5$. From these figures, one sees that, at fixed $\pi T$, increasing $b$ leads to decreasing $x / x_{\mathrm{SYM}}$, indicating that the stopping distance decreases with the increase in $b$. Therefore, one concludes that the inclusion of back reaction decreases the stopping distance thus enhancing the energy loss of light quarks, consistently with the findings of the drag force [21] and the jet quenching parameter [22]. Also, by comparing the two figures, one finds that the higher is the temperature, the smaller the absolute value of the slope, which means the back reaction has a stronger effect on the stopping distance (or energy loss) at low temperature, in accord with [21,22] as well.

\subsection{Shooting string}

In this section, we study the energy loss of light quarks within the finite-endpoint-momentum shooting string approach, or in the short shooting string approach $[18,19]$, Therein, one considers a particular type of classical string motion: the string endpoint starts near the horizon and then shoots toward the boundary, carrying some energy and momentum which is gradually bled off into the rest of the string during its rise. 
Therefore, this motion is termed finite-endpoint-momentum shooting string motion.

Now we follow the argument in $[18,19]$ to study the effect of back reaction on the energy loss of light quarks for the background metric (13). The instantaneous energy loss is

$\frac{\mathrm{d} E}{\mathrm{~d} x}=-\frac{|L|}{2 \pi \alpha^{\prime}} \frac{1}{z^{2}}$,

where $L$ denotes the null geodesics that the endpoint follows. From the above expression one finds that a small $z$ (which means that the endpoint starts near the boundary) will yield a large energy loss, implying the jets will be quenched quickly and will not be observable. To avoid this situation, the strings need to start close to the horizon.

Here again, one considers the quark moving along the $x_{3}$ direction. Also, for convenience, we take $R=1$. The energy and momentum of the quark are conserved as

$E=-\frac{1}{\eta} \frac{f(z)}{z^{2}}$

and

$p_{x_{3}}=\frac{1}{\eta z^{2}} \frac{\mathrm{d} x}{\mathrm{~d} t}$,

where $\eta$ represents the auxiliary field. Given that, the null geodesics reads

$L=\frac{E}{p_{x_{3}}}=-f(z) \frac{\mathrm{d} t}{\mathrm{~d} x}$.

The finite-momentum endpoints will move along $d s^{2}=0$, leading to

$$
\left(\frac{\mathrm{d} x}{\mathrm{~d} z}\right)^{2}=\frac{1}{L^{2}-f(z)} .
$$

If the endpoints follow the null geodesics, the denominator of (28) will vanish at $z=z_{*}$, resulting in

$L=-\sqrt{f\left(z_{*}\right)}$,

which leads to

$\frac{\mathrm{d} x}{\mathrm{~d} z}=\frac{1}{\sqrt{f\left(z_{*}\right)-f(z)}}$.

Finally, one arrives at the energy loss for the back reacted gravity background,

$\frac{\mathrm{d} E}{\mathrm{~d} x}=-\frac{1}{2 \pi \alpha^{\prime}} \frac{\sqrt{f\left(z_{*}\right)}}{z^{2}}$.

To find how the energy loss depends on $b$ and on $x$, one needs to solve the null geodesics equation (30). Note that it is hard to solve (30) analytically, but it is possible numerically. The numerical procedures are as follows: 1 . Sending $z_{*} \rightarrow 0$ and then, for a given value of $b$, one can numerically integrate (30) and invert to get $z(x)$. 2. Plugging $z(x)$ into (31) one can obtain $\mathrm{d} E / \mathrm{d} x$ as a function of $x$ and $b$.
Before proceeding, we recall the results of $\mathcal{N}=4 \mathrm{SYM}$ [19], given by

$$
\left(\frac{\mathrm{d} E}{\mathrm{~d} x}\right)_{\mathrm{SYM}}=-\frac{\pi \sqrt{\lambda}}{2} T^{2}(1+\pi T x)^{2},
$$

where $T=1 / \pi z_{h}$ and $\lambda=g_{\mathrm{YM}}^{2} N_{c}=\frac{1}{\alpha^{\prime 2}}$. One sees that, for small, intermediate and large $x, \mathrm{~d} E / \mathrm{d} x$ looks like $T^{2}, x T^{3}$ and $x^{2} T^{4}$, respectively.

Let us discuss the results. In the left panel of Fig. 2, we plot $(\mathrm{d} E / \mathrm{d} x) /(\mathrm{d} E / \mathrm{d} x)_{S Y M}$ against $b$ with fixed temperature, e.g., $\pi T=0.5$, for two different values of $x$, where the top is for $x=0.1$ while the bottom is for $x=0.01$. From these figures, one can see that increasing $b$ leads to increasing $(\mathrm{d} E / \mathrm{d} x) /(\mathrm{d} E / \mathrm{d} x)_{S Y M}$, indicating that the inclusion of back reaction increases the energy loss, in agreement with the analysis of the stopping distance from the previous section and also consistent with findings of the drag force [21] and jet quenching parameter [22]. Moreover, by comparing the two figures, one finds that the bigger $x$ is, the larger the slope of the curve, implying $b$ has stronger effect for large $x$.

Also, in the right panel of Fig. 2, we plot $(\mathrm{d} E / \mathrm{d} x) /$ $(\mathrm{d} E / \mathrm{d} x)_{\mathrm{SYM}}$ against $b$ with fixed $x=0.1$ for two values of $\pi T$, where the top is for $\pi T=0.5$ while the bottom is for $\pi T=1$. It is seen that with the increase in $T$, the slope of the curve decreases, indicating that $b$ has a stronger effect on the energy loss at low temperature, in agreement with the findings of the stopping distance. The physical interpretation of the results will be discussed in the next section.

\section{Conclusion and discussion}

Using the AdS/CFT correspondence, we studied the effect of back reaction on the energy loss of a light quark in a strongly coupled $\mathcal{N}=4$ SYM plasma within the falling string and shooting string approaches, respectively. The back reaction considered here comes from the presence of static heavy quarks uniformly distributed over the SYM plasma. The corresponding back reaction effect is modeled by means of the deformation of the geometry due to a finite density string cloud. For the falling string case, we computed the stopping distance of an image jet induced by a massless source field, characterized by a massless particle falling along the null geodesic and found that the inclusion of back reaction decreases the stopping distance thus enhancing the energy loss. Meanwhile for the shooting string case, we investigated the instantaneous energy loss using the finite-endpointmomentum shooting string approach and observed that the presence of back reaction increases the energy loss. For both cases, it is found that the back reaction effect is more pronounced at low temperature. All these results are in agreement with the findings of the drag force [21] and jet quench- 

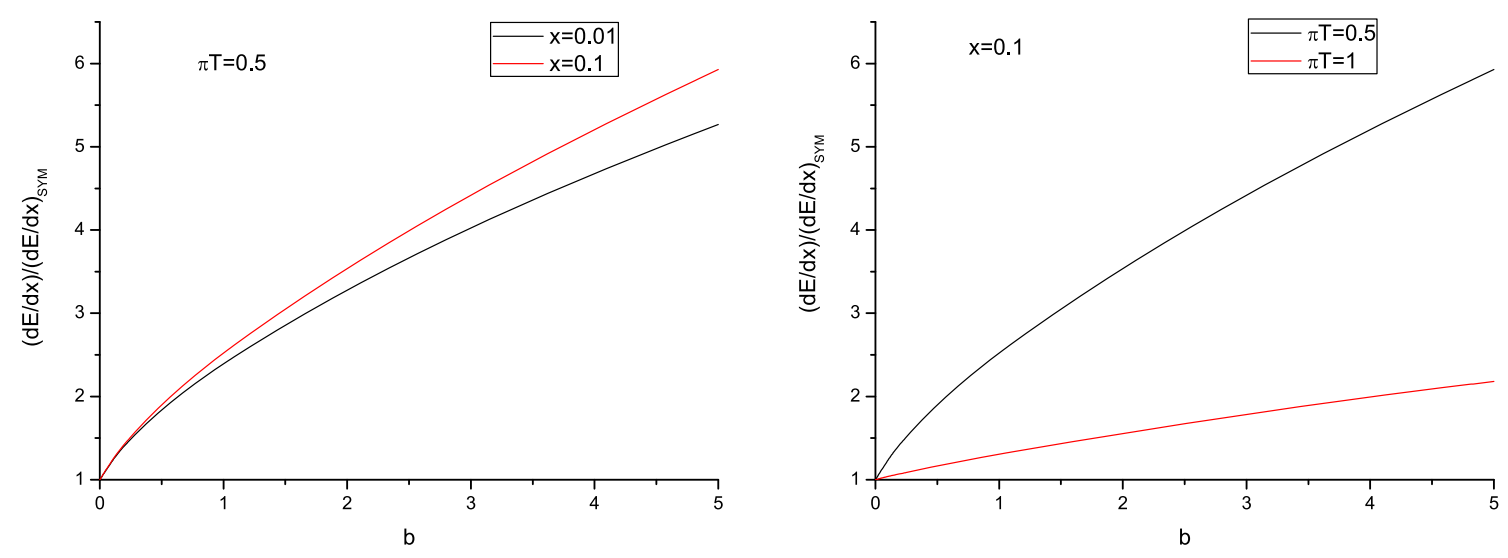

Fig. $2(\mathrm{~d} E / \mathrm{d} x) /(\mathrm{d} E / \mathrm{d} x)_{a}$ versus $b$

ing parameter [22] analyses. Going one step further, one may draw the conclusion that the effects of back reaction on the energy loss of heavy quarks and light quarks are consistent: the presence of back reaction enhances the quark energy loss.

However, there are some drawbacks with this model. First, it is a major simplification of the real physical system (the heavy quarks in homogeneous approximation might not be consistent with reality), but the hope is that it captures some of the physics to provide useful insight. Moreover, it is conformal, different from QCD. Then considering such an effect in non-conformal systems would also be instructive.

Acknowledgements This work is supported by the NSFC Grant Nos. 11735007, 11890711.

Data Availability Statement This manuscript has no associated data or the data will not be deposited. [Authors' comment: This is a theoretical study and no experimental data has been listed.]

Open Access This article is licensed under a Creative Commons Attribution 4.0 International License, which permits use, sharing, adaptation, distribution and reproduction in any medium or format, as long as you give appropriate credit to the original author(s) and the source, provide a link to the Creative Commons licence, and indicate if changes were made. The images or other third party material in this article are included in the article's Creative Commons licence, unless indicated otherwise in a credit line to the material. If material is not included in the article's Creative Commons licence and your intended use is not permitted by statutory regulation or exceeds the permitted use, you will need to obtain permission directly from the copyright holder. To view a copy of this licence, visit http://creativecomm ons.org/licenses/by/4.0/.

Funded by SCOAP ${ }^{3}$.

\section{References}

1. M. Connors, C. Nattrass, R. Reed, S. Salur, Rev. Mod. Phys. 90, 025005 (2018)

2. G.Y. Qin, X.-N. Wang, Int. J. Mod. Phys. E 24(11), 1530014 (2015)

3. E.V. Shuryak, Prog. Part. Nucl. Phys. 53, 273 (2004)

4. E.V. Shuryak, Nucl. Phys. A 750, 64 (2005)

5. J.M. Maldacena, Adv. Theor. Math. Phys. 2, 231 (1998)

6. S.S. Gubser, I.R. Klebanov, A.M. Polyakov, Phys. Lett. B 428, 105 (1998)

7. E. Witten, Adv. Theor. Math. Phys. 2, 253 (1998)

8. J.C. Solana, H. Liu, D. Mateos, K. Rajagopal, U.A. Wiedemann, arXiv: 1101.0618

9. C.P. Herzog, A. Karch, P. Kovtun, C. Kozcaz, L.G. Yafe, JHEP 07, 013 (2006)

10. S.S. Gubser, Phys. Rev. D 74, 126005 (2006)

11. H. Liu, K. Rajagopal, U.A. Wiedemann, Phys. Rev. Lett. 97, 182301 (2006)

12. H. Liu, K. Rajagopal, U.A. Wiedemann, JHEP 03, 066 (2007)

13. S.S. Gubser, D.R. Gulotta, S.S. Pufu, F.D. Rocha, JEHP 10, 052 (2008)

14. P.M. Chesler, K. Jensen, A. Karch, Phys. Rev. D 79, 025021 (2009)

15. P.M. Chesler, K. Jensen, A. Karch, L.G. Yaffe, Phys. Rev. D 79, 125015 (2009)

16. P. Arnold, D. Vaman, JHEP 10, 099 (2010)

17. P. Arnold, D. Vaman, JHEP 04, 027 (2011)

18. A. Ficnar, S.S. Gubser, Phys. Rev. D 89, 026002 (2014)

19. A. Ficnar, S.S. Gubser, M. Gyulassy, Phys. Lett. B 738, 464 (2014)

20. L.G. Yaffe, P.M. Chesler, Phys. Rev. Lett. 99, 152001 (2007)

21. S. Chakrabortty, Phys. Lett. B 705, 244 (2011)

22. S. Chakrabortty, Tanay K. Dey, JHEP 05, 094 (2016)

23. S. Chakrabortty, S. Pant, K. Sil, JHEP 06, 061 (2020)

24. P. Wu, X. Zhu, Z. Zhang, Nucl. Phys. B 952, 114917 (2020)

25. Z. Zhang, Phys. Rev. D 101, 106005 (2020) 